\title{
A MEASURE OF MUTUAL DIVERGENCE AMONG A NUMBER OF PROBABILITY DISTRIBUTIONS
}

\author{
J.N. KAPUR \\ Department of Mathematics \\ Indian Institute of Technology \\ New Delhi, India 110016 \\ VINOD KUMAR and UMA KUMAR \\ School of Business \\ Carleton University \\ Ottawa, Canada K1S 5B6 \\ (Received September 11, 1985 and in revised form September 22, 1986)

\begin{abstract}
The principle of optimality of dynamic programming is used to prove three major inequalities due to Shannon, Renyi and Holder. The inequalities are then used to obtain some useful results in information theory. In particular measures are obtained to measure the mutual divergence among two or more probability distributions.
\end{abstract}

KEY WORDS AND PHRASES. Divergence measures, dynamic programming, Shannon, Renyi and Holder inequalities.

1980 AMS SUBJECT CLASSIFICATION CODE. 94A17, 94A15, 90C50, 90C39

\section{INTRODUCTION}

An important problem in business, economic and social sciences is concerned with measuring differences in specific characteristics of business, economic or social groups. For example, we may like to compare the balance sheets of $m$ business firms, or economic development of $\boldsymbol{m}$ nations or the difference in opinions of $\boldsymbol{m}$ directors of a company or the population compositions of $m$ cities and 80 on.

Let $q_{i j}$ represent the share of the jth item from the ith group. Thus $q_{i j}$ may represent proportionate contributions of the jth item in the balance sheet of the ith company or it may represent the population of persons in the jth income group of the ith nation or the proportionate allotment to the jth item of the budget propoeed by the ith director or the proportionate population of the jth social group in the ith city and so on. Let

$$
Q_{i}=\left(q_{i 1}, q_{i 2}, \cdots, q_{i n}\right) ; i=1,2, \cdots, m
$$


where

$$
\sum_{i=1}^{n} q_{i j}=1, \quad q_{i j} \geq 0
$$

Given $Q_{1}, Q_{2}, \cdots, Q_{m}$, our problem is to find how different $Q_{1}, Q_{2}, \cdots, Q_{m}$ are. We can regard $Q_{1}, Q_{2}, \cdots, Q_{m}$ as $m$ probability distributions and thus our problem is to find a measure of mutual divergence between $Q_{1}, Q_{2}, \cdots, Q_{m}$.

This measure should have the following properties:

(i) It should depend on $Q_{1}, Q_{2}, \cdots, Q_{m}$

(ii) It should be a continuous function of all $q_{i j}$ 's

(iii) It should not change when $q_{i 1}, q_{i 2}, \cdots, q_{i n}$ are permuted among themselves, provided the same permutation is used for each of $Q_{1}, Q_{2}, \cdots, Q_{m}$.

(iv) It should be always $\geq 0$

(v) It should vanish when $Q_{1}=Q_{2}=\cdots=Q_{m}$

(vi) It should vanish only when $Q_{1}=Q_{2}=\cdots=Q_{m}$

(vii) It should possibly be a convex function of $Q_{1}, Q_{2}, \cdots, Q_{m}$

For two distributions, $(m=2)$ we have a number of measures of directed divergence due to Kullback-Leibler, Havrda-Charvat, Renyi, Kapur and others [5]. However, in real life we are concerned with $m(\geq 2)$ distributions. We can of course find directed divergence between every pair of $m$ distributions and then find some sort of an average of these directed divergences.

However, we shall prefer to have a unified measure depending directly on the $m$ distributions. We develop such a measure in the present paper.

Since one important condition for the measure is non-negativity and this condition is expressed as an inquality, an important role is played in the development of our new measure by the special inequalities due to Shannon, Renyi, Holder and their generalizations. We give alternate proofs of all these inqualities by using dynamic programming and then use these inequalities to develop our new measure.

\section{DYNAMIC PROGRAMMING AND INEQUALITIES}

Kapur [4] used dynamic programming technique of Bellman [2] to show that the maximum value of $\sum_{i=1}^{n} p_{i} \ln p_{i}$ subject to $p_{1} \geq 0, p_{2} \geq 0,0, \cdots, p_{n} \geq 0, \sum_{i=1}^{n} p_{1}=c$ is $-c \ln (c / n)$ and it arises when $p_{1}=p_{2}=\cdots=p_{n}=c / n$. In particular, when $c=1$, we find that the maximum value of the Shannon's [7] entropy measure $-\sum_{i=1}^{n} p_{i} \ln p_{i}$ subject to $p_{1} \geq 0, p_{2} \geq 0, \cdots, p_{n} \geq 0, \sum_{i=1}^{n} p_{i}=1$ is $\ln n$ and arise when all the probabilities are equal. The result is equivalent to showing that

$$
-\sum_{i=1}^{n} p_{i} \ln p_{i} \leq \ln n
$$

for all probability distributions. 
The same technique can be used to establish inequalities of the form

$$
\sum_{i=1}^{n} f\left(p_{i}, q_{i}\right) \leq A \text { or } \sum_{i=1}^{n} f\left(p_{i}, q_{i}\right) \geq B
$$

In the first case, we show that the maximum value of $\sum_{i=1}^{n} f\left(p_{i}, q_{i}\right)$ is $A$ and in the second case, we show that the minimum value of $\sum_{i=1}^{n} f\left(p_{i}, q_{i}\right)$ is $B$.

Suppose we have to maximize $\sum_{i=1}^{n} f\left(p_{i}, q_{i}\right)$ subject to

$$
p_{1} \geq 0, p_{2} \geq 0, \cdots, p_{n} \geq 0 ; q_{1} \geq 0, q_{2} \geq 0, \cdots, q_{n} \geq 0, \sum_{i=1}^{n} p_{i}=a, \sum_{i=1}^{n} q_{i}=b
$$

Let the maximum value be $g_{n}(a, b)$.

We may keep $p_{1}, p_{2}, \cdots, p_{n}$ fixed'and vary only $q_{1}, q_{2}, \cdots, q_{n}$ subject to $\sum_{i=1}^{n} q_{i}=b$, then the principle of optimality gives

$$
g_{n}(a, b)=\max _{0 \leq q_{1} \leq b}\left[f\left(p_{1}, q_{1}\right)+g_{n-1}\left(a-p_{1}, b-q_{1}\right)\right]
$$

Since $p_{1}$ is fixed, we have to maximize a function of one variable $q_{1}$ only. Equation (2.4) give a recurrence relation which along with the knowledge of $f_{1}(a, b)$ can enable us to obtain $g_{n}(a, b)$ for all values of $n$, by proceeding step by step.

The same procedure can be used to find the minimum value.

\section{SHANNON'S INEQUALITY}

Here

$$
f\left(p_{i}, q_{i}\right)=p_{i} \ln \frac{p_{i}}{q_{i}}
$$

This is a convex function of $q_{i}$ and we seek to determine the minimum value of $\sum_{i=1}^{n} f\left(p_{i}, q_{i}\right)$.

We get

$$
g_{1}(a, b)=a \ln \frac{a}{b}
$$

Using (2.4)

$$
\begin{aligned}
g_{2}(a, b) & =\max _{0 \leq q_{1} \leq b}\left[p_{1} \ln \frac{p_{1}}{q_{1}}+\left(a-p_{1}\right) \ln \frac{a-p_{1}}{b-q_{1}}\right] \\
& =a \ln \frac{a}{b}
\end{aligned}
$$

Assuming

$$
g_{n}(a, b)=a \ln \frac{a}{b}
$$

(2.4) gives

$$
\begin{aligned}
g_{n+1}(a, b) & =\min _{0 \leq q_{1} \leq b}\left[p_{1} \ln \frac{p_{1}}{q_{1}}+\left(a-p_{1}\right) \ln \frac{a-p_{1}}{b-q_{1}}\right] \\
& =a \ln \frac{a}{b}
\end{aligned}
$$

Hence by mathematical induction we get

$$
\min \sum_{i=1}^{n} p_{i} \ln \frac{p_{i}}{q_{i}}=a \ln \frac{a}{b}
$$


or

$$
\sum_{i=1}^{n} p_{i} \ln \frac{p_{i}}{q_{i}} \geq a \ln \frac{a}{b}
$$

The minimum value is obtained when

$$
\frac{p_{1}}{q_{1}}=\frac{p_{2}}{q_{2}}=\cdots=\frac{p_{n}}{q_{n}}=\frac{a}{b}
$$

If $a=b=1$, then we get Shannon's inequality

$$
\sum_{i=1}^{n} p_{i} \ln \frac{p_{i}}{q_{i}} \geq 0 \text { and } \sum_{i=1}^{n} p_{i} \ln \frac{p_{i}}{q_{i}}=0 \text { iff } p_{i}=q_{i} \text { for each } i
$$

If $b \leq a$,

$$
\sum_{i=1}^{n} p_{i} \ln \frac{p_{i}}{q_{i}} \geq a \ln \frac{a}{b} \geq 0
$$

Alternative proofs are given in Aczel and Daroczy [1].

\section{RENYI'S INEQUALITY}

Here

$$
\begin{aligned}
& f\left(p_{i}, q_{i}\right)=\frac{1}{\alpha-1}\left(p_{i}^{\alpha} q_{i}^{1-\alpha}-p_{i}\right) \\
& g_{1}(a, b)=\frac{1}{\alpha-1}\left(a^{\alpha} b^{1-\alpha}-a\right)
\end{aligned}
$$

$f\left(p_{i}, q_{i}\right)$ is a convex function of $q_{i}$ and we try to find the minimum value of $\sum_{i=1}^{n}\left(p_{i}, q_{i}\right)$

$$
\begin{aligned}
g_{2}(a, b) & =\min _{0 \leq q_{1} \leq b}\left[\frac{1}{\alpha-1}\left(p_{1}^{\alpha} q_{1}^{1-\alpha}-p_{1}\right)+\frac{1}{\alpha-1}\left(a-p_{1}\right)^{\alpha}\left(b-q_{1}\right)^{1-\alpha}-\left(a-p_{1}\right)\right] \\
& =\frac{1}{\alpha-1}\left(a^{\alpha} b^{1-\alpha}-a\right) .
\end{aligned}
$$

By using mathematical induction, we can show that

$$
\frac{1}{\alpha-1}\left[\sum_{i=1}^{n} p_{i}^{\alpha} q_{i}^{1-\alpha}-\sum_{i=1}^{n} p_{i}\right] \geq \frac{1}{\alpha-1}\left[a^{\alpha} b^{1-\alpha}-a\right]
$$

If $a=b=1$, we get Renyi's inequality

$$
\frac{1}{\alpha-1}\left[\sum_{i=1}^{n} p_{i}^{\alpha} q_{i}^{1-\alpha}-1\right] \geq 0
$$

The inequality (4.5) will hold whenever $a=b$, even if the common value is not unity.

Alternative proofs of this inequality are available in Aczel and Daroczy [1] and Kapur $[6]$.

\section{HOLDER'S INEQUALITY}

Let $f_{n}(M)$ denote the minimum value of 


$$
U=\left(\sum_{j=1}^{n} x_{j}^{p}\right)^{q / p}\left(\sum_{j=1}^{n} y_{j}^{q}\right),
$$

where $x_{j}$ 's and $y_{j}$ 's are positive real numbers, $x_{j}$ 's are fixed and $y_{j}$ 's vary subject to $\sum_{\jmath=1}^{n} x, y_{j}=M$, then

$$
\begin{gathered}
f_{1}(M)=M^{q} \\
f_{2}(M)=\left(x_{1}^{p}+x_{2}^{p}\right)^{q / p} \min _{0} \leq z_{1} \leq M\left[\left(\frac{z_{1}}{x_{1}}\right)^{q}+\left(\frac{M-z_{1}}{x_{2}}\right)^{q}\right]
\end{gathered}
$$

Now

$$
\begin{aligned}
g\left(z_{1}\right)=\left(\frac{z_{1}}{x_{1}}\right)^{q}+\left(\frac{M-z_{1}}{x_{2}}\right)^{q} & \Rightarrow g^{\prime}\left(z_{1}\right)=q\left(\frac{z_{1}^{q-1}}{x_{1}^{q}}-\frac{\left(M-z_{1}\right)^{q-1}}{x_{2}^{q}}\right) \\
& \Rightarrow g^{\prime \prime}\left(z_{1}\right)=q(q-1)\left(\frac{z_{1}^{q-2}}{x_{1}^{q}}+\frac{\left(M-z_{1}\right)^{q-2}}{x_{2}^{q}}\right)
\end{aligned}
$$

If $q(q-1)>0$, the minimum value of $g\left(z_{1}\right)$ occurs when

$$
\frac{z_{1}}{x_{1}^{q / q(q-1)}}=\frac{M-z_{1}}{x_{2}^{q /(q-1)}}=\frac{M}{x_{1}^{q /(q-1)}+x_{2}^{q /(q-1)}}
$$

and

$$
\begin{aligned}
{\left[g\left(z_{1}\right)\right]_{\min } } & =M^{q} /\left[x_{1}^{q /(q-1)}+x_{2}^{q /(q-1)}\right]^{q-1} \\
& =M^{q} /\left[x_{1}^{p}+x_{2}^{p}\right]^{q-1}
\end{aligned}
$$

If

$$
\frac{1}{p}+\frac{1}{q}=1 \text { or } q-1=q / p
$$

then

$$
f_{2}(M)=\left(x_{1}^{p}+x_{2}^{p}\right)^{q / p} \frac{M^{q}}{\left(x_{1}^{p}+x_{2}^{p}\right)^{q / p}}=M^{q}
$$

If we assume that $f_{n}(M)=M^{q}$, then the principle of optimality gives

$$
\begin{aligned}
f_{n+1}(M) & =\left(\sum_{i=1}^{n} x_{i}^{p}\right)^{q / p} \min _{0 \leq z_{1} \leq M}\left[\left(\frac{z_{1}}{x_{1}}\right)^{q}+\frac{\left(M-z_{1}\right)^{q}}{\left(x_{n}^{p}+\cdots+x_{n+1}^{p}\right)^{q / p}}\right] \\
& =M^{q}
\end{aligned}
$$

thus if $q(q-1)>0$,

$$
\left(\sum_{j=1}^{n} x_{j}^{p}\right)^{q / p}\left(\sum_{j=1}^{n} y_{j}^{q}\right) \geq M^{q}
$$

If $q>1$, this gives

$$
\sum_{j=1}^{n} x_{j} y_{j} \leq\left(\sum_{j=1}^{n} x_{j}^{p}\right)^{1 / p}\left(\sum_{j=1}^{n} y_{j}^{q}\right)^{1 / q}
$$

Using (5.10), we find that the inequality (5.12) holds if $q>1, p>1, p^{-1}+q^{-1}=1$.

If $q<0,0<p<1$ or $o<q<1$ and $p<0$ and $p^{-1}+q^{-1}=1$, then the inequality in (5.12) is reversed and we get 


$$
\sum_{j=1}^{n} x_{j} y_{j} \leq\left(\sum_{j=1}^{n} x_{j}^{p}\right)^{1 / p}\left(\sum_{j=1}^{n} y_{j}^{q}\right)^{1 / q} .
$$

\section{A GENERALIZATION OF HOLDER'S INEQUALITY}

Now we consider the minimization of

$$
V_{n}=\left(\sum_{j=1}^{n} x_{j}^{p}\right)^{r / p}\left(\sum_{j=1}^{n} y_{j}^{q}\right)^{r / q}\left(\sum_{j=1}^{n} z_{j}^{r}\right)
$$

where $x_{j}$ 's, $y_{j}$ 's, $z_{j}$ 's are all pooitive real numbers, $x_{j}$ 's and $y_{j}$ 's are kept fixed and $z_{j}$ 's vary subject to

$$
\sum_{j=1}^{n} x_{j} y_{j} z_{j}=M
$$

If $k_{n}(M)$ is the minimum value of $U$, then

$$
\begin{gathered}
k_{1}(M)=M^{r} \\
k_{2}(M)=\left(\sum_{j=1}^{2} x_{j}^{p}\right)^{r / p}\left(\sum_{j=1}^{2} y_{j}^{q}\right)^{r / q} 0 \leq u \leq M\left[\left(\frac{u}{x_{1} y_{1}}\right)^{r}+\left(\frac{M-u}{x_{2} y_{2}}\right)^{r}\right] \\
=\left(\sum_{j=1}^{2} x_{j}^{p}\right)^{r / p}\left(\sum_{j=1}^{2} y_{j}^{q}\right)^{r / p}\left[\frac{M^{r}}{\left(x_{1} y_{1}\right)^{r /(r-1)}+\left(x_{2} y_{2}\right)^{r /(r-1)}}\right]
\end{gathered}
$$

provided $r>1$, so that in this case

$$
V_{2} \geq M^{r}\left(\sum_{j=1}^{2} x_{j}^{p}\right)^{r / p}\left(\sum_{j=1}^{2} y_{j}^{q}\right)^{r / q}\left[\left(x_{1} y_{1}\right)^{r /(r-1)}+\left(x_{2} y_{2}\right)^{r /(r-1)}\right]^{-(r-1)}
$$

Similarly when $r>1$

$$
V_{n} \geq M^{r}\left(\sum_{j=1}^{n} x_{j}^{p}\right)^{r / p}\left(\sum_{j=1}^{n} y_{j}^{q}\right)^{r / q}\left[\sum_{j=1}^{n}\left(x_{j} y_{j}\right)^{r /(r-1)}\right]^{-(r-1)}
$$

Now we consider the case when $p>1, q>1, r>1, p^{-1}+q^{-1}+r^{-1}=1$, so that

$$
\frac{1}{p}+\frac{1}{q}=\frac{r-1}{r} \text { or } \frac{r}{r-1} \frac{1}{p}+\frac{r}{r-1} \frac{1}{q}=1
$$

or

$$
\frac{1}{p^{\prime}}+\frac{1}{q^{\prime}}=1 ; p^{\prime}=p \frac{r-1}{r}, q^{\prime}=q \frac{r-1}{r}
$$

Again by Holder's inequality proved in the last section when $p^{\prime}>1, q^{\prime}>1$

$$
\left(\sum_{j=1}^{n} u_{j}^{p^{\prime}}\right)^{1 / p^{\prime}}\left(\sum_{j=1}^{n} v_{j}^{q^{\prime}}\right)^{1 / q^{\prime}} \geq \sum_{j=1}^{n} u_{j} v_{j}
$$


or

$$
\left(\sum_{j=1}^{n} u_{j}^{\frac{n(r-1)}{r}}\right)^{\pi(r-1)}\left(\sum_{j=1}^{n} v_{j}^{\frac{r(r-1)}{r}}\right)^{\frac{r}{r-1)}} \geq \sum_{j=1}^{n} u_{j} v_{j}
$$

or

$$
\left(\sum_{j=1}^{n} x_{j}^{p}\right)^{r / p}\left(\sum_{j=1}^{n} y_{j}^{q}\right)^{r / q} \geq\left(\sum_{j=1}^{n} x_{j}^{\frac{r}{r-1}} y_{j}^{\frac{r}{r-1}}\right)^{r-1}
$$

From (6.6) and (6.8), $V_{n} \geq M^{r}$ or

$$
\left(\sum_{j=1}^{n} x_{j}^{p}\right)^{1 / p}\left(\sum_{j=1}^{n} y_{j}^{q}\right)^{1 / q}\left(\sum_{j=1}^{n} z_{j}^{r}\right)^{1 / r} \geq \sum_{j=1}^{n} x_{j} y_{j} z_{j}
$$

whenever (6.7) is satisfied.

The method of proof is quite general and (6.9) is easily generalized to give

$$
\left(\sum_{j=1}^{n} x_{j 1}^{q 1}\right)^{1 / q_{1}}\left(\sum_{j=1}^{n} x_{j 2}^{q 2}\right)^{1 / q_{2}} \cdots\left(\sum_{j=1}^{n} x_{j m}^{q_{m}}\right)^{1 / q_{m}} \geq \sum_{j=1}^{n} x_{j 1} x_{j 2} \cdots x_{j m}
$$

whenever

$$
q_{1}>1, q_{2}>1, \cdots, q_{m}>1 ; q_{1}^{-1}+q_{2}^{-1}+\cdots+q_{m}^{-1}=1
$$

Inequality (6.10) can also be written as

$$
\sum_{j=1}^{n} y_{j 1}^{\alpha_{1}} y_{j 2}^{\alpha_{2}} \cdots y_{j m}^{\alpha_{m}} \leq\left(\sum_{j=1}^{n} y_{j 1}\right)^{\alpha_{1}}\left(\sum_{j=1}^{n} y_{j 2}\right)^{\alpha_{2}} \cdots\left(\sum_{j=1}^{n} y_{j m}\right)^{\alpha_{m}}
$$

whenever

$$
0<\alpha_{1}<1,0<\alpha_{2}<2, \cdots, 0<\alpha_{m}<1 ; \alpha_{1}+\alpha_{2}+\cdots+\alpha_{m}=1
$$

If $y_{j i}$ 's represent probabilities, we get

$$
\sum_{j=1}^{n} p_{j 1}^{\alpha_{1}} p_{j 2}^{\alpha_{2}} \cdots p_{j m}^{\alpha_{m}} \leq 1
$$

In particular for $m=2,(6.14)$ gives

or

$$
\sum_{j=1}^{n} p_{j}^{\alpha} q_{j}^{1-\alpha} \leq 1 \text { when } 0<\alpha<1
$$

$$
(\alpha-1)^{-1}\left(\sum_{j=1}^{n} p_{j}^{\alpha} q_{j}^{1-\alpha}-1\right) \geq 0
$$

Alternative proofs of Holder's inequality are given in Hardy, Littlewood and Polya [3] and Marshall and Olkin [7].

7. HOLDER'S GENERALIZED INEQUALITY FOR OTHER VALUES OF PARAMETERS 
Holder's generalized inequality (6.10) holds whenever $q_{1}, q_{2} \cdots, q_{n}$ satisfy (6.11). However, if (6.11) are not satisfied, the inequality sign in (6.10) may be reversed or no inequality may hold.

Thus for the special case $m=3$ if $0<r<1$, the inequality sign in (6.5) is reversed. If one of $p$ or $q$ is negative, then in Holder's inequality also the sign is reversed so that if $0<r<1$, one of $p$ and $q$ is negative and $p^{-1}+q^{-1}+r^{-1}=1$, then

$$
\left(\sum_{j=1}^{n} x_{j}^{p}\right)^{1 / p}\left(\sum_{j=1}^{n} y_{j}^{q}\right)^{1 / q}\left(\sum_{j=1}^{n} z_{j}^{r}\right)^{1 / r} \leq \sum_{j=1}^{n} x_{j} y_{j} z_{j}
$$

while if $r>1, p>1, q>1$ and $p^{-1}+q^{-1}+r^{-1}=1$, then inequality (6.9) holds. If $p^{-1}+q^{-1}+r^{-1}=1$, but neither of the two sets of conditions is satisfied, then neither (6.9) nor (7.1) may hold. Thus

$$
\begin{aligned}
& \text { if } p=2, q=4, r=4(6.9) \text { holds } \\
& \text { if } p=1 / 2, q=-1 / 3, r=1 / 2,(7.1) \text { holds } \\
& \text { if } p=-2, q=-2, r=1 / 2 \text {, neither (6.9) nor (7.1) holds. }
\end{aligned}
$$

Similarly given $q_{1}, q_{2}, \cdots, q_{n}$ we can find whether $(6.10)$ holds or whether $(6.10)$ with reversed sign of inequality holds or whether neither of these two inequalities holds.

\section{APPLICATIONS TO INFORMATION THEORY}

From Renyi's or Holder's inequality, it follows that

$$
D_{\alpha}(P: Q)=\frac{1}{\alpha-1}\left(\sum_{i=1}^{n} p_{i}^{\alpha} q_{i}^{1-\alpha}-1\right) \geq 0, \alpha \neq 1, \alpha>0
$$

where $P=\left(p_{1}, p_{2}, \cdots, p_{n}\right), Q=\left(q_{1}, q_{2}, \cdots, q_{n}\right)$ are two probability distributions. Also $D_{\alpha}(P: Q)=0$ iff $p_{i}=q_{i}$ for each $i$. In the limiting case when $\alpha \rightarrow 1$, this gives

$$
D_{1}(P: Q)=\sum_{i=1}^{n} p_{i} \ln \frac{p_{i}}{q_{i}} \geq 0
$$

$D_{\alpha}(P: Q)$ or $D_{1}(P: Q)$ can be used as a measure of directed divergence of $P$ from $Q$.

for these distributions, we similarly have from generalized Holder's inequality, that

$$
D_{\alpha, \beta}(P: Q: R)=\frac{1}{\alpha^{2}+\beta^{2}-1}\left[\sum_{i=1}^{n} p_{i}^{\alpha} q_{i}^{\beta} r_{i}^{1-\alpha-\beta}-1\right] \geq 0
$$

whenever $0 \leq \alpha \leq 1,0 \leq \beta \leq 1, \alpha+\beta<1$, and it vanishes iff $p_{i}=q_{i}=r_{i}$ for each $i$.

If $\alpha=0$, it measures the directed divergence of $Q$ from $P$

$\beta=0$, it measures the directed divergence of $P$ from $R$ and if

$\alpha+\beta=1$, it measures the directed divergence of $P$ from $Q$.

Collectively $D_{\alpha, \beta}(P: Q: R)$ can be used as a measure of mutal divergence among three distributions. 
Generalizing we get the measure

$$
\begin{aligned}
& D_{\alpha_{1}, \alpha_{2}, \cdots, \alpha_{m}}\left(P_{1}: P_{2}: \cdots: P_{m}\right) \\
& =\frac{1}{\alpha_{1}^{2}+\alpha_{2}^{2}+\cdots \alpha_{m}^{2}-1}\left[\sum_{i=1}^{n} p_{i 1}^{\alpha_{1}} p_{i 2}^{\alpha_{2}} \cdots p_{i m}^{\alpha_{m}}-1\right] \geq 0
\end{aligned}
$$

where $0<\alpha_{1}<1,0<\alpha_{2}<1, \cdots, 0<\alpha_{m}<1, \alpha_{1}+\alpha_{2}+\cdots \alpha_{m}=1$. By putting some of $\alpha_{1}, \alpha_{2}, \cdots, \alpha_{m}$ equal to zero, it can also be used to measure all divergences among subsets of $P_{1}, P_{2}, \cdots, P_{m}$.

for three distributions, Theil [9] had proposed the measure

$$
D(P: Q: R)=\sum_{i=1}^{n} p_{i} \ln \frac{r_{i}}{q_{i}}
$$

as a measure of information improvement. However, this can be both negative and positive. On the other hand our measure (8.3) is always $\geq 0$ and can be a more effective measure of improvement.

The measure (8.3) and (8.4) are also recursive since

$$
D_{\alpha, \beta, n}(P: Q: R)=\frac{1}{\alpha^{2}+\beta^{2}-1}\left[\sum_{i=1}^{n} p_{i}^{\alpha} q_{i}^{\beta} r_{1}^{1-\alpha-\beta}-1\right]
$$

or

$$
\begin{aligned}
\left(\alpha^{2}+\beta^{2}-1\right) D_{\alpha, \beta, n}(P: Q: R) & =p_{1}^{\alpha} q_{1}^{\beta} r_{1}^{1-\alpha-\beta}+p_{2}^{\beta} q_{2}^{\beta} r_{2}^{1-\alpha-\beta} \\
& -\left(p_{1}+p_{2}\right)^{\alpha}\left(q_{1}+q_{2}\right)^{\beta}\left(r_{1}+r_{2}\right)^{1-\alpha-\beta} \\
& +\left(\alpha^{2}+\beta^{2}-1\right) D_{\alpha, \beta, n-1}\left(p_{1}, p_{2}, p_{3}, \cdots, p_{n} ;\right. \\
& \left.q_{1}+q_{2}, q_{3}, \cdots, q_{n} ; r_{1}+r_{2}, r_{3}, \cdots, r_{n}\right)
\end{aligned}
$$

or

$$
\begin{aligned}
& D_{\alpha, \beta, n}\left(p_{1}, \cdots, p_{n} ; q_{1}, \cdots, q_{n} ; r_{1}, \cdots, r_{n}\right) \\
& =D_{\alpha, \beta, n-1}\left(p_{1}+p_{2}, p_{3}, \cdots, p_{n} ; q_{1}+q_{2}, q_{3}, \cdots, q_{n} ; r_{1}+r_{2}, r_{3}, \cdots, r_{n}\right) \\
& \quad+\left(p_{1}+p_{2}\right)^{\alpha}\left(q_{1}+q_{2}\right)^{\beta}\left(r_{1}+r_{2}\right)^{1-\alpha-\beta} . \\
& \quad H_{2}\left(\frac{p_{1}}{p_{1}+p_{2}}, \frac{p_{2}}{p_{1}+p_{2}}, \frac{q_{1}}{q_{1}+q_{2}}, \frac{q_{2}}{q_{1}+q_{2}}, \frac{r_{1}}{r_{1}+r_{2}}, \frac{r_{2}}{r_{1}+r_{2}}\right)
\end{aligned}
$$

\section{MEASURE OF SYMMETRIC MUTUAL DIVERGENCE}

The measure (8.3) is not symmetric in the sense that

$$
D_{\alpha, \beta}(P: Q: R), D_{\alpha, \beta}(Q: P: R), D_{\alpha, \beta}(P: R: Q)
$$

etc. are not equal unless $\alpha=\beta=1 / 3$. The measure

$$
D_{\frac{1}{3}, \frac{3}{3}}(P: Q: R)=\frac{9}{7}\left[1-\sum_{i=1}^{n} p_{i}^{1 / 3} q_{i}^{1 / 3} r_{i}^{1 / 3}\right]
$$

is symmetric. Another symmetric measure of mutal divergence is given by

$$
\begin{aligned}
D_{\alpha, \beta}(P: Q: R) & +D_{\alpha, \beta}(P: R: Q)+D_{\alpha, \beta}(Q: P: R) \\
& +D_{\alpha, \beta}(Q: R: P)+D_{\alpha, \beta}(R: Q: P)
\end{aligned}
$$


The corresponding symmetric measure for $m$ distributions will be the sum of $m !$ mutual divergences.

This is a two-parameter family of measures of mutal symmetric divergences.

The measure (9.2) can be compared with the measure

$$
D_{\alpha}(P: Q)+D_{\alpha}(Q: P)+D_{\alpha}(P: R)+D_{\alpha}(R: P)+D_{\alpha}(Q: R)+D_{\alpha}(R: Q)
$$

which is also a measure of symmetric mutal divergence.

\section{CONCLUDING REMARKS}

By using dynamic programming, it can be easily shown that the maximum value of $x_{1}, x_{2} \cdots x_{n}$ subject to $x_{1}+x_{2}+\cdots+x_{n}=c, x_{1} \geq 0, \cdots, x_{n} \geq 0$ is $(c / n)^{n}$ and the minimum value of $x_{1}+x_{2}+\cdots+x_{n}$ subject to $x_{1} x_{2} \cdots x_{n}=1, x_{1}>0, x_{2}>0, \cdots, x_{n}>0$ is $n^{n} \sqrt{d}$. From either of these results, the Arithmetic-Geometric-Mean Inequality viz.

$$
\frac{x_{1}+x_{2}+\cdots+x_{n}}{n} \geq \sqrt{x_{1} x_{2} \cdots x_{n}}
$$

can be deduced. From this inequality, one can obtain Shannon's and Holder's inequalities. One can also prove Renyi's entropy first by usine dvnamic oronramming and then deduce Shannon's inequality from it. One can also prove first Holder's inequality, by using dynamic programming and then deduce Renyi's Shannon's and AGM inequalities from it. In a sense all these inequalities are equivalent.

Holder's, Renyi's and Shannon's inequality enable us to get a number of measure of directd divergence between two given distributions and with the help of these measures of directed divergence. We can obtain a number of messures of entropy and inaccuracy.

The generalized Holder's inequality enable us to get a number of measures of mutal divergence between $m(>2)$ probability distributions.

\section{ACKNOWLFDGEMENT}

This research was supported in part by the Natural Sciences and Engineering Research Council of Canada.

\section{REFERENCES}

1. ACZEL, G. and DAROCZY, Z. On Measures of Information and Their Characterization, Academic Press, New York, 1975.

2. BELLMAN, R. and DREYFUS, S.E., Appliod Dynamic Programming, 1962.

3. HARDY, G.H., LITTLEWOOD and POLYA, G. Inequalities, Cambridge University Press, 1952. 
4. KAPUR, J.N. On Some Applications of Dynamic Programming to Information Theory, Proc. Ind. Acad. Sci. , 68A (1968) 1-11.

5. KAPUR, J.N. A Comparative Assessment of Measure of Directed Divergence, Adv. Manag. Studies, 5 (1984) 1-16.

6. KAPUR, J.N. Convex Semi-Metric Spaces and Generalized Shannon's Inequalities. Ind. Journ. Pure Appl. Math. , (to appear), 1986.

7. MARSHALL, A.W., and OLKIN, I. Inequalities Theory of Majorisation and its Applications, Academic Press, New York, 1979.

8. SHANNON, C.E. A Mathematical Theory of Communication, Bell System Technical J. , 27 (1948), 379-423, 623-656.

9. THEIL, H. Economics and Information Theory, North Holland, Amsterdam, 1967. 


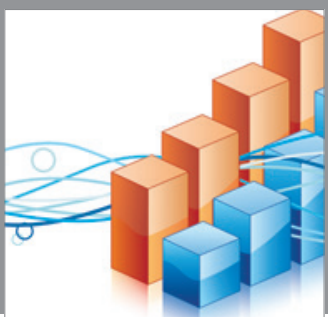

Advances in

Operations Research

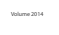

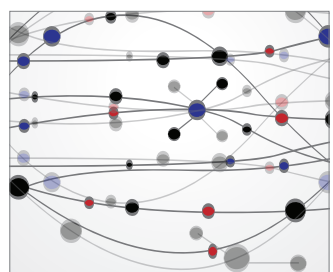

\section{The Scientific} World Journal
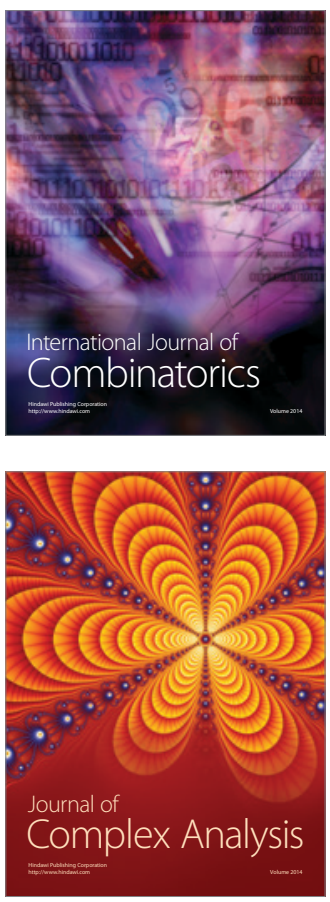

International Journal of

Mathematics and

Mathematical

Sciences
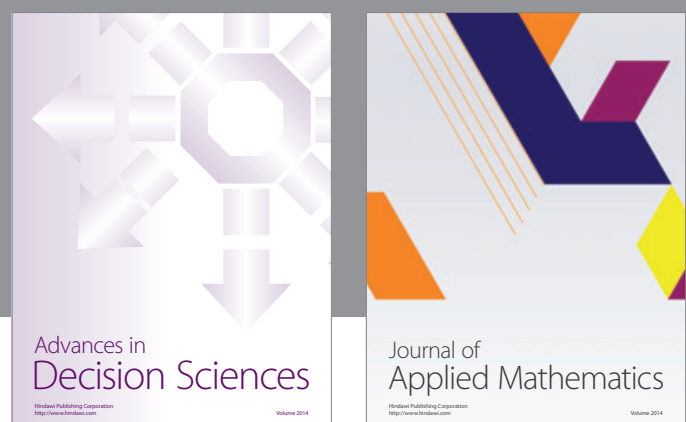

Journal of

Applied Mathematics
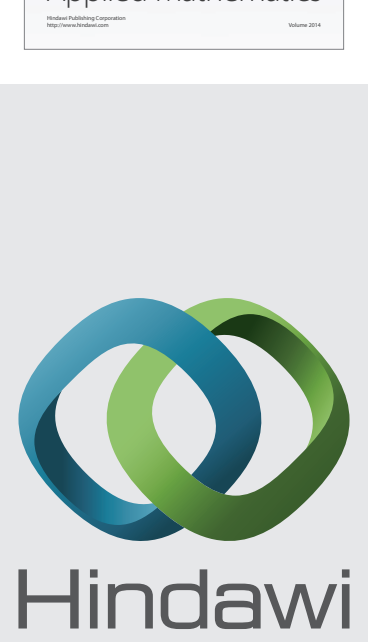

Submit your manuscripts at http://www.hindawi.com
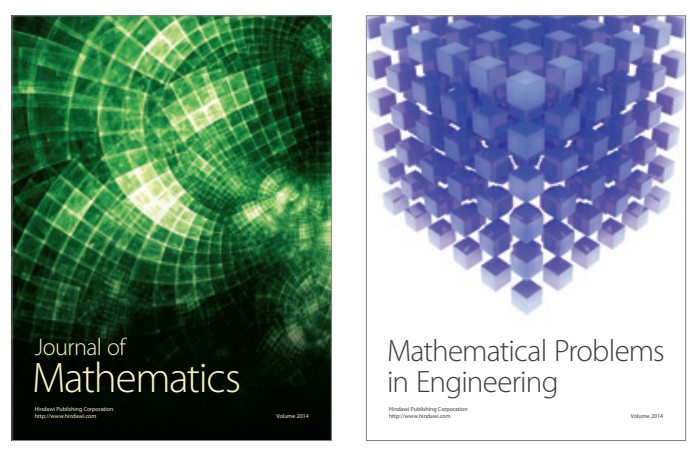

Mathematical Problems in Engineering
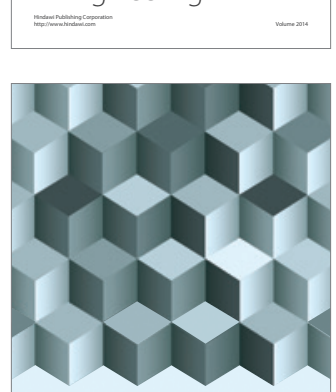

Journal of

Function Spaces
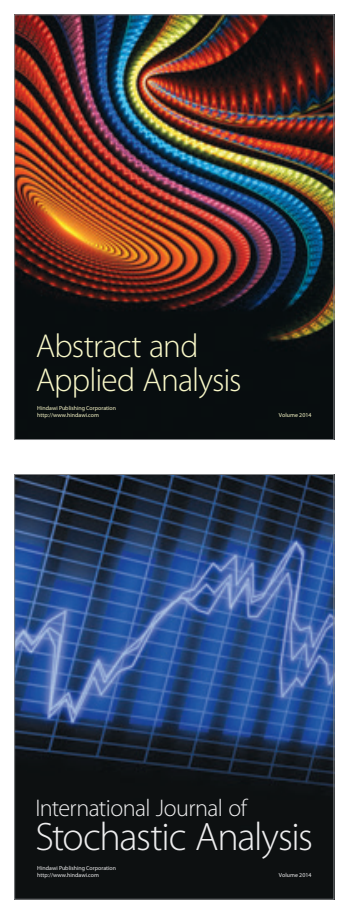

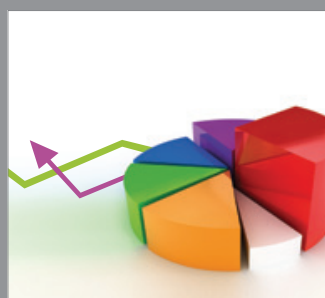

ournal of

Probability and Statistics

Promensencen
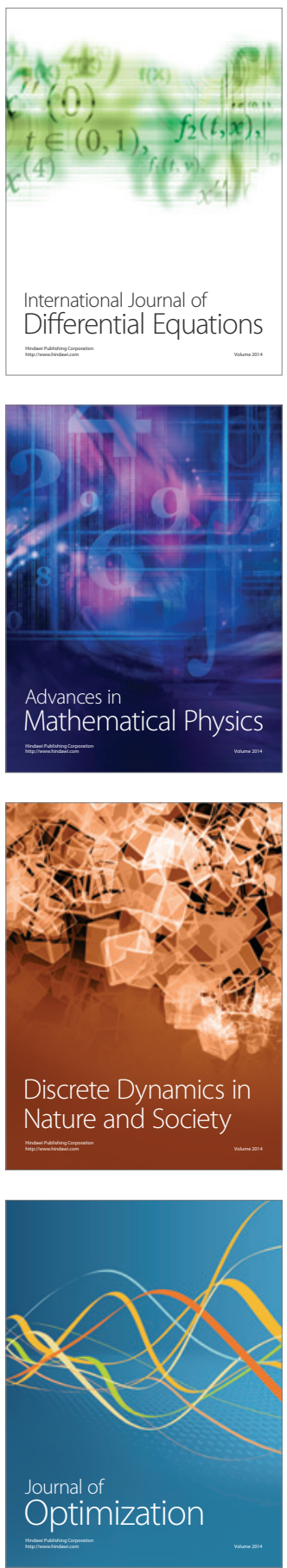